\title{
Correcting the Influence of an Asymmetric Line Spread Function in 2-Degree Field Spectrograph Data
}

\author{
Nicolas F. Martin ${ }^{\mathrm{A}, \mathrm{D}}$, Rodrigo A. Ibata ${ }^{\mathrm{A}}$, Blair C. Conn ${ }^{\mathrm{B}}$, Mike J. Irwin ${ }^{\mathrm{C}}$, \\ and Geraint F. Lewis ${ }^{\mathrm{B}}$ \\ A Observatoire de Strasbourg, 11 rue de l'Université, 67000 Strasbourg, France \\ ${ }^{B}$ Institute of Astronomy, School of Physics, A29, University of Sydney, Sydney NSW 2006, Australia \\ ${ }^{\mathrm{C}}$ Institute of Astronomy, Madingley Road, Cambridge, CB3 0HA, UK \\ D Corresponding author. Email: martin@astro.u-strasbg.fr
}

Received 2005 March 11, accepted 2005 May 24

\begin{abstract}
We investigate the role of asymmetries in the line spread function of the 2-degree field (2dF) spectrograph and the variations in these asymmetries with the CCD, the plate, the time of observation, and the fibre. A data-reduction pipeline is developed that takes these deformations into account for the calibration and cross-correlation of the spectra. We show that, using the emission lines of calibration lamp observations, we can fit the line spread function with the sum of two Gaussian functions representing the theoretical signal and a perturbation of the system. This model is then used to calibrate the spectra and generate templates by downgrading high-resolution spectra. Thus, we can cross-correlate the observed spectra with templates degraded in the same way. Our reduction pipeline is tested on real observations and provides a significant improvement in the accuracy of the radial velocities obtained. In particular, the systematic errors that were as high as $\sim 20 \mathrm{~km} \mathrm{~s}^{-1}$ when applying the AAO reduction package 2DFDR are now reduced to $\sim 5 \mathrm{~km} \mathrm{~s}^{-1}$. Even though the $2 \mathrm{dF}$ spectrograph is to be decommissioned at the end of 2005, the analysis of archival data and previous studies could be improved by the reduction procedure we propose here.
\end{abstract}

Keywords: instrumentation: spectrograph — techniques: spectroscopic — galaxies: kinematics and dynamics

\section{Introduction}

The 2-degree field (2dF) multi-object spectrograph at the Anglo-Australian Telescope can observe up to 400 objects within a $2 \mathrm{dF}$ on the sky through two different spectrograph settings at the same time (Lewis et al. 2002) ${ }^{1}$. Each spectrograph accepts light from 200 fibres, which are positioned by a robot on a plate in the focal plane of the telescope, to produce spectra of low to medium resolution depending on the chosen grating. Two full sets of fibres on separate plates ensure that re-configuring can be done in parallel with observing, hence minimizing time lost to the positioning of fibres during the night.

With its high-resolution gratings, $2 \mathrm{dF}$ spectrograph should be an ideal instrument for studies of the kinematics of resolved stars; however, it has been shown that radial velocities obtained using the $2 \mathrm{dF}$ spectrograph are plagued by systematic errors of the order of $10-20 \mathrm{~km} \mathrm{~s}^{-1}$ (Cannon 2002; Stanford \& Cannon 2002). While known, these systematic errors are not easily corrected for they are not constant over all the observations. Observations performed on different CCDs or plates, during different nights or at different times during the same night, can

${ }^{1}$ See also www . aao.gov.au/2df/ show variations in radial velocity that are worse than the $5 \mathrm{~km} \mathrm{~s}^{-1}$ internal precision the $2 \mathrm{dF}$ should achieve.

The most probable culprit for these variations is a change in the point spread function of the system across the CCDs due to the optics of the cameras, tilts in the slit blocks, or changes in the focus of the spectrographs with temperature or orientation of the telescope, given that they are mounted on the top of the AAT close to the dome aperture (Cannon 2002). These deformations produce an asymmetric line spread function (LSF) on the extracted spectra which can be revealed by artificially asymmetric emission/absorption lines.

Our group recently undertook a radial velocity survey of the region around the Canis Major (CMa) dwarf galaxy and other nearby overdensities in Red Giant Branch stars (RGBs) and Red Clump stars (Martin et al. 2004, 2005). Given its high number of targets on a wide field of view, the $2 \mathrm{dF}$ spectrograph is currently the best instrument for such a study. However, one of the key features of accretion streams is their very low dispersion in radial velocities. Thus, it is crucial that we achieve the lowest possible systematic errors on the velocities of our sample stars to be as close as possible to the internal dispersion of a population. Moreover, for the case of the CMa study, the velocity of the constituent stars is close to those of the thin disc 
(Martin et al. 2004). In the regions where the CMa population is significantly contaminated by disc stars, a systematic error of $10-20 \mathrm{~km} \mathrm{~s}^{-1}$ could diffuse the signal of the extra-galactic population and could prevent detection.

For these reasons, we constructed a reduction pipeline for $2 \mathrm{dF}$ observations that takes into account the asymmetry of the LSF of the extracted spectra and corrects it. Since the shape of the LSF plays a role in the calibration of the observed spectra and their cross-correlation with templates to obtain radial velocities, our pipeline works as follows:

1. determination of the deformations of the LSF across the CCD using the emission lines of the observed calibration lamps;

2. use of the determined model of the LSF to calibrate the arc spectra;

3. use of the determined model of the LSF to generate template spectra (from high-resolution spectra) deformed in the same way as the observed spectra;

4. cross-correlation of the observed spectra with the obtained template spectra.

In Section 2, we describe the different steps of the pipeline, while in Section 3 the derived radial velocities are compared to reference observations and we analyze the accuracy of the new velocities. Section 4 summarizes the pipeline and the results.

In the following, the $2 \mathrm{dF}$ observations are first corrected for the flat field and extracted using the $2 \mathrm{dF}$ data reduction package (2DFDR) provided by the AAO (Taylor et al. 1996) $)^{2}$. Sky subtraction is also performed using the 2DFDR package.

\section{Observations and Reduction}

In this section, the observations on which we apply our reduction pipeline are part of our CMa radial velocity survey. We targeted the Red Giant Branch stars of a field centred on $l=240^{\circ}, b=-8.8^{\circ}$. We employed two different spectrograph settings, with the $1200 \mathrm{~V}$ grating on spectrograph 1 (covering 4600-5600 $\AA$ at $1 \AA$ per pixel, these are the CCD1 data) and with the $1200 \mathrm{R}$ grating on spectrograph 2 (covering 8000-9000 A, also at $1 \AA$ per pixel, these are the CCD2 data).

Stanford \& Cannon (2002) showed that observations made with CCD2 are better than those obtained through CCD1. We will therefore concentrate of correcting the deformations of the LSF on CCD1. This is also justified by our analysis of CCD2 observations (see Section 2.5).

\subsection{The LSF Model}

For a given optical system, the output signal $s_{0}(\lambda)$ that is observed at wavelength $\lambda$ can be expressed as

$$
s_{\mathrm{o}}(\lambda)=\left(s_{\mathrm{i}} * G\right)(\lambda)
$$

where $s_{\mathrm{i}}$ is the input of the system and $G$ is the LSF of the system. When observed on a CCD, each pixel $X$, of

\footnotetext{
${ }^{2}$ See also www . aao.gov. au/2df/software.html\#2dfdr
}

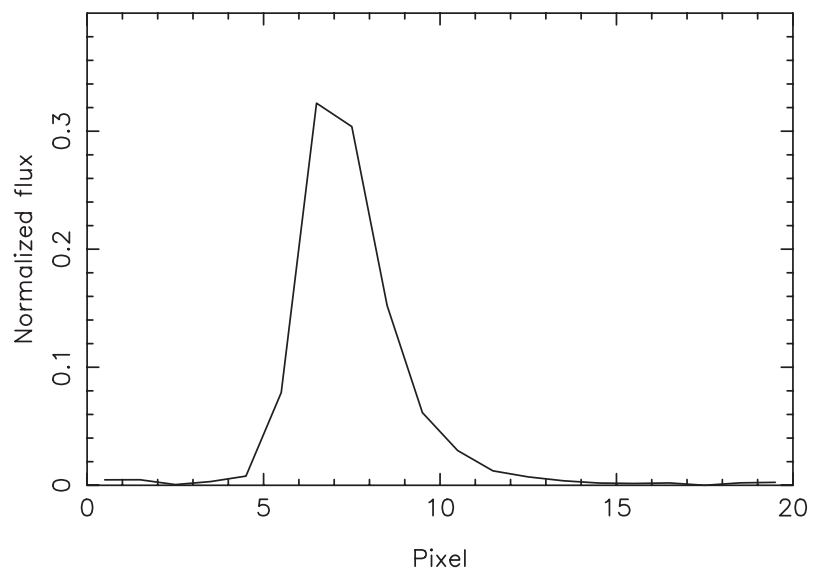

Figure $15105.541 \AA \mathrm{Cu}$ emission line extracted from fibre 1 of a CCD1 calibration lamp observation. The emission line shows an asymmetry towards higher wavelengths that accounts for $\sim 30 \%$ of the total signal.

width $S \AA$, receives part of this output signal, and has a value $F(X)$ such that

$$
F(X)=\int_{X-0.5 \cdot S}^{X+0.5 \cdot S} s_{\mathrm{o}}(\lambda) \mathrm{d} \lambda .
$$

When observing an emission line (EL) centred on $\mu_{1}$, the input signal is

$$
s_{\mathrm{i}}(\lambda)=\delta\left(\lambda-\mu_{1}\right)
$$

which produces the observed signal

$$
s_{\mathrm{o}}(\lambda)=\int_{-\infty}^{\infty} G(\tau) \delta\left(\lambda-\mu_{1}-\tau\right) \mathrm{d} \tau=G\left(\lambda-\mu_{1}\right)
$$

This means that the signal, $F(X)$, produced by this EL on pixel $X$ of the $2 \mathrm{dF}$ with grating $1200 \mathrm{~V}$ - for which $S=1 \AA-$ is

$$
F(X)=\int_{X-0.5}^{X+0.5} G\left(\lambda-\mu_{1}\right) \mathrm{d} \lambda .
$$

If the system through which the observations are performed was perfect, the LSF of the system $(G)$ would be a symmetric Gaussian function with a dispersion that depends on the quality of the system. However, this is not the case for the $2 \mathrm{dF}$ spectrograph. As can be seen in Figure 1 for an EL produced by the observation of a copper-argon calibration lamp on CCD1, the LSF is deformed and generates an EL with an asymmetric wing at higher wavelengths. This deformation is significant and can account for as much as $30 \%$ of the total signal of the EL.

To model the deformed LSF, we represent it as the sum of two Gaussian functions. The first one, $G_{1}$, is the signal that would be observed through a perfect system and is only determined by the dispersion of the would be perfect system: $\sigma_{1}$. The second Gaussian function, $G_{2}$, is considered as a perturbation of the system that produces the deformation. It is shifted by $\Delta \mu$ compared to $G_{1}$, has its 
own dispersion $\sigma_{2}$, and contains a fraction $A$ of the total signal. Hence this model of the LSF can be expressed by

$$
\begin{aligned}
G(\lambda)= & (1-A) G_{1}(\lambda)+A G_{2}(\lambda) \\
= & \frac{1-A}{\sqrt{2 \pi} \sigma_{1}} \exp \left[-\frac{1}{2}\left(\frac{\lambda}{\sigma_{1}}\right)^{2}\right] \\
& +\frac{A}{\sqrt{2 \pi} \sigma_{2}} \exp \left[-\frac{1}{2}\left(\frac{\lambda-\Delta \mu}{\sigma_{2}}\right)^{2}\right]
\end{aligned}
$$

With this model and using Equation (5), the value $F(X)$ of pixel $X$ of an emission line becomes

$$
\begin{aligned}
& F(X) \\
& =\frac{1-A}{\sqrt{2 \pi} \sigma_{1}} \int_{X-0.5}^{X+0.5} \exp \left[-\frac{1}{2}\left(\frac{\lambda-\mu_{1}}{\sigma_{1}}\right)^{2}\right] \mathrm{d} \lambda \\
& +\frac{A}{\sqrt{2 \pi} \sigma_{2}} \int_{X-0.5}^{X+0.5} \exp \left[-\frac{1}{2}\left(\frac{\lambda-\left(\mu_{1}-\Delta \mu\right)}{\sigma_{2}}\right)^{2}\right] \mathrm{d} \lambda
\end{aligned}
$$

\subsection{Fitting the Model}

As expressed in Equation (7), the shape of an EL at a given position on the CCD is only determined by five free parameters:

- $\mu_{1}$ : the theoretical centre of the emission line;

- $\sigma_{1}$ : the standard deviation of the theoretical, symmetric LSF;

- $A$ : the fraction of the total signal that is in the perturbation;

- $\sigma_{2}$ : the standard deviation of the perturbation;

- $\Delta \mu$ : the shift between the two Gaussian functions $G_{1}$ and $G_{2}$. Since the asymmetry of the emission lines is to higher wavelengths, $\Delta \mu>0$.

$\mu_{1}$ is the only free parameter that depends on the EL while the other four $\left(A, \sigma_{1}, \sigma_{2}\right.$, and $\left.\Delta \mu\right)$ define the shape of the LSF for the considered fibre, time and plate. By fitting this model $F$ of the EL on the EL observed on the CCD, $\widetilde{F}$ (such as in Figure 1$)^{3}$, we can deduce the parameters that define the LSF of the system for the fibre, wavelength, time, and plate of the observation.

To completely characterize the deformations of the LSF, it would be necessary to define these parameters in both the $X$ (wavelength) and $Y$ (fibre number) directions of the CCD. However, since for our observations the region we use to cross-correlate the spectra and the templates is near the centre of the wavelength range of the CCD and since there are few ELs in this region, we model only the deformations of the LSF in the $Y$ direction of the CCD. Yet, observations performed with different settings may require a complete two-dimensional model of the LSF.

\footnotetext{
${ }^{3}$ In the following, the ${ }^{\sim}$ functions (e.g. $\widetilde{F}$ ) represent the observed data while the functions without the ${ }^{\sim}$ represent the model to be fitted to the data (e.g. F).
}

Among the $\sim 10$ ELs that are observed with the $1200 \mathrm{~V}$ grating with a copper-argon calibration lamp, the best choice to fit the model $F$ is the $\mathrm{Cu}$ line at $5105.541 \AA$ since it is the strongest line and it is at the centre of the region we will use to cross-correlate the spectra and the templates (the $4800 \AA$ to $5250 \AA$ region) $)^{4}$. We could determine the five free parameters of the model on the $\widetilde{F}$ function of each fibre; however, the low number of pixels over which $\widetilde{F}(X) \gtrsim 0.0$ (less than 20 , see Figure 1) would produce high uncertainties on the parameters. Therefore, we choose to fit the same LSF model on up to ten consecutive fibres (depending on the presence of dead fibres) at the same time. Indeed, the spectrograph is constructed with fibres coming in blocks of ten, which means the fibres in each of these blocks should have analogous $\mathrm{LSF}^{5}$ but changes may appear between each block.

By extracting the EL of all the fibres and concatenating them, we generate a new function $\widetilde{F}^{\prime}$ that is modelled by $F^{\prime}$ :

$$
F^{\prime}(X)=\sum_{i=1}^{N} \int_{X-0.5}^{X+0.5} G\left(\lambda-\mu_{i}\right) \mathrm{d} \lambda .
$$

In this new model, $G$ is still the LSF model as expressed in Equation (6), $\mu_{i}$ is the centre of the $i$-th EL, and $N$ is the number of fibres in the group (i.e. ten minus the number of dead fibres). This concatenated model $F^{\prime}$ now contains $4+N$ free parameters: the $N$ centres of the ELs $\left(\mu_{i}\right)_{1 \leq i \leq N}$ and the four parameters of the $\operatorname{LSF}\left(A, \sigma_{1}, \sigma_{2}\right.$ and $\Delta \mu)$. This time, however, there are $20 \times N$ points to adjust these $4+N$ free parameters, which ensures lower uncertainties on the estimate of the parameters.

Each group of fibres $(1-10,11-20, \ldots)$ is processed as follows:

- for each fibre

- check if fibre is not dead;

- extract the $\mathrm{Cu}$ line at 5105.541 $\AA$ (around pixel 500) to generate $\widetilde{F}$;

- concatenate all the fibres of the group to generate $\widetilde{F}^{\prime}$;

- fit model $F^{\prime}(4+N$ parameters $)$ to data $\widetilde{F}^{\prime}$.

When extracting the EL, we take care to extract approximately the same region around the line for all the fibres, even if their centre varies with the position across the CCD. We detect the pixel $X_{\mathrm{C}}$ with the highest value of the EL and extract six pixels before $X_{\mathrm{C}}$ and 13 pixels after $X_{\mathrm{C}}$ to account for the asymmetry to higher wavelength in the shape of the EL. We then correct from a possible continuum by subtracting a linear fit between the average value of the first three pixels of $\widetilde{F}$ and the average value of the last three pixels. Finally, to correct differences in the response

\footnotetext{
${ }^{4}$ We choose this region for the cross-correlation with the templates because it is free of strong sky features. It is also located at the centre of the CCD along the wavelength axis, which should diminish potential asymmetries of the LSF generated along this axis.

5 This was tested by taking the fibres in smaller groups. The results are similar, but with higher uncertainties on the values of the parameters.
} 
of the fibres, we normalize each EL so that

$$
\int \widetilde{F}(X) \mathrm{d} X=1.0
$$

An example of the final data $\widetilde{F}^{\prime}$ we obtain after the concatenation of the fibres of a group is shown as hollow circles in Figure 2.

To fit the model $F^{\prime}$ to the data $\widetilde{F}^{\prime}$, we use the MRQMIN routine described in Press et al. (1992) and determine the $4+N$ parameters. Since determining all the parameters at the same time proves difficult, we determine independently the $N$ EL-related parameters $\left(\mu_{i}\right)_{1 \leq i \leq N}$ and the four LSF-related parameters $A, \sigma_{1}, \sigma_{2}$, and $\Delta \mu$. The fitting procedure is as follows:

(1) The $N \mu_{i}$ values are determined for a loose grid of fixed values of $A, \sigma_{1}, \sigma_{2}$, and $\Delta \mu$. The $\left(\mu_{i}\right)_{1<i<N}$ producing the lowest $\chi^{2}$ (in the sense of MRQMIN) are used as input values.

(2) The $\left(\mu_{i}\right)_{1 \leq i \leq N}$ are fixed and the four LSF-related parameters are determined.

(3) These LSF-related parameters are fixed to their best values and used as input values to re-determine the $\left(\mu_{i}\right)_{1 \leq i \leq N}$.
(4) These are once again fixed to re-determine the best values of $A, \sigma_{1}, \sigma_{2}$, and $\Delta \mu$.

Steps (3) and (4) are used as a 'sanity check' to verify the independence of the two sets of parameters. Since $\chi^{2}$ is reduced by less than $1 \%$ between step (2) and step (4), our hypothesis can be taken as valid and the two sets of parameters determined independently. Figure 2 shows the fit resulting from this procedure as a thick line. It is visibly good with a well-determined position for the ELs (the different $\mu_{i}$ ) and it reproduces the asymmetric shape of the ELs with a large wing at higher pixel number.

For a given calibration lamp observation, this procedure is repeated for the 20 groups of up to ten valid fibres. An example of the evolution of the four LSF-related parameters is shown in Figure 3. The first striking feature is that the four parameters evolve across the CCD, which means that the asymmetry of the LSF is not constant for the different fibres of the same observation run. The most important parameter, $A$, which represents the weight of the perturbation goes up to $\sim 30 \%$ at the edges of the CCD but is as low as $\sim 15 \%$ at its centre. If this behaviour is expected (Cannon 2002), the deformations at the edges of the CCD are so important they can easily explain strong systematic

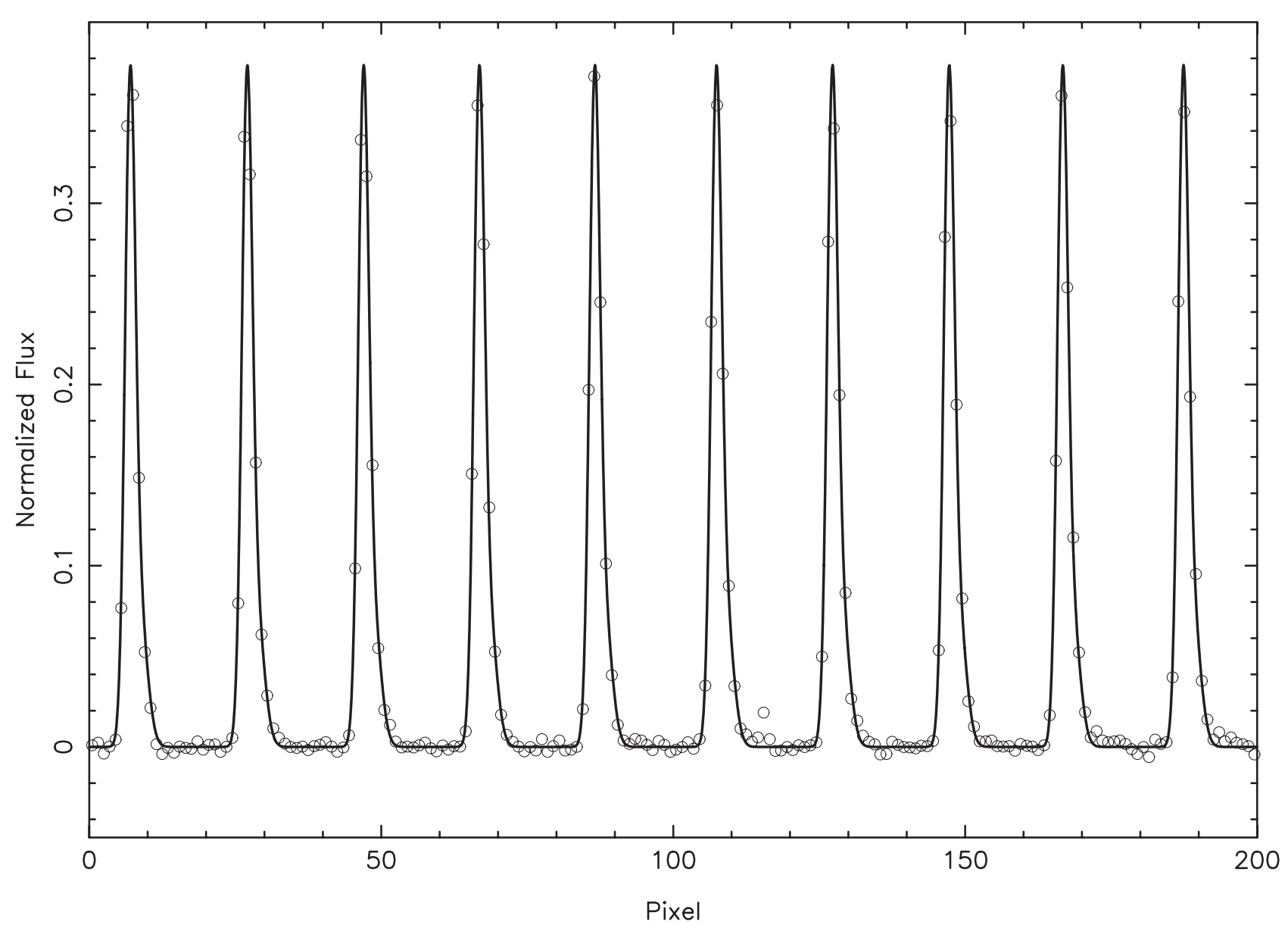

Figure 2 Concatenation of emission lines from a group of fibres (hollow circles, corresponding to the $\widetilde{F}^{\prime}$ function). Since there are no dead fibres in this group, ten emission lines were extracted and were used for the fit following the procedure described in the text. The best fit (the $F^{\prime}$ function) is shown as a thick line and faithfully represents the data. In particular, it reproduces the asymmetric, high-wavelength wing of each emission line. 
errors in the radial velocities if they are not accounted for. All the parameters have a roughly symmetric evolution with values at the two edges of the CCD being in the same range and different from those in the centre.

One of the assumptions in our LSF model is that it can be divided into a 'theoretical signal' $G_{1}$ (determined by $\sigma_{1}$ ) and a 'perturbation' $G_{2}$ (determined by $A, \Delta \mu$, and $\sigma_{2}$ ) that creates the asymmetry. This seems to be confirmed by the almost constant value of $\sigma_{1}$ that only evolves between 0.81 and 0.85 over the CCD, while at the same time the 'perturbation dispersion', $\sigma_{2}$, is in a range that is one order of magnitude higher (between 1.3 and 1.7). Moreover, the value of $\sigma_{1}$ is similar to what is found on the symmetric ELs observed on CCD2 (see Section 2.5).
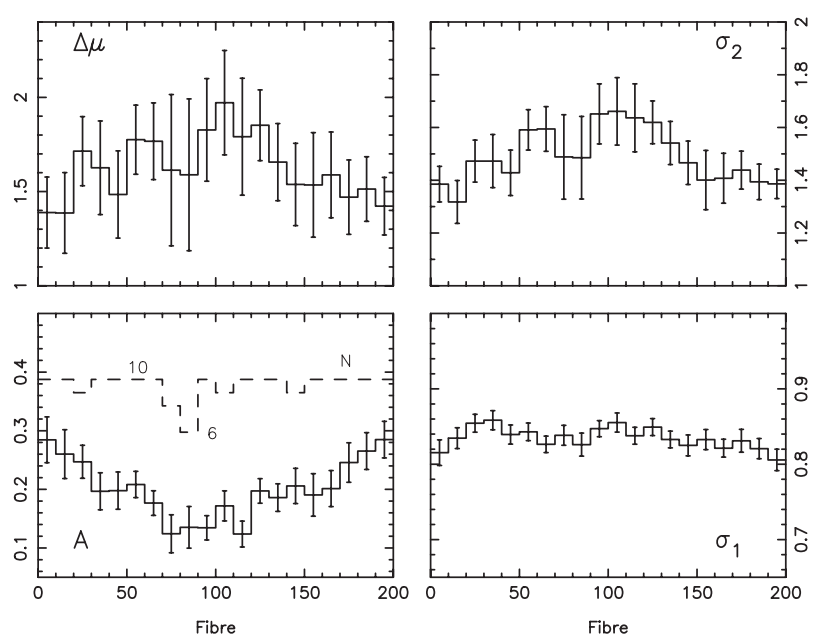

Figure 3 Evolution of the four LSF-related parameters $A$ (bottom-left panel), $\sigma_{1}$ (bottom right), $\sigma_{2}$ (top right), and $\Delta \mu$ (top left) across CCD1. The three parameters representing the perturbation of the $\operatorname{LSF}\left(A, \sigma_{2}\right.$, and $\left.\Delta \mu\right)$ show substantial variations across the CCD. The number of fibres, $N$, used for the fit is plotted as a thin dashed histogram in the bottom-left panel.
Though the parameters evolve in the expected way, a more detailed look at their behaviour shows many gaps and spikes where one would expect a smoother evolution over the CCD. This is partly due to changes in the number of fibres used to fit the model $(N$, dashed histogram in the bottom-left panel of Figure 3): for instance, there are four dead fibres between fibre 81 and fibre 90 , so, in the corresponding group, there remains only six ELs for the fit. For this group, the parameters $\Delta \mu$ and $\sigma_{2}$ show a gap and have wider error bars. These gaps and spikes are also explained by the shallow $\chi^{2}$ space which provides many slightly different LSF models with small differences in $\chi^{2}$.

Another interesting point to look at is the evolution of the asymmetry on the LSF during a night of observation and the influence of the plate on which the observations are done. Figure 4 shows the evolution of the four parameters of the LSF for the seven observation runs we made on 2004 April 9. For the same plate, the results are similar, with parameters that are really identical (e.g. $\sigma_{1}$ and $A$ ) but the parameters of the 'perturbation' tend to vary, even though they show a similar behaviour. Likewise, even if the deformation of the LSF evolves in a similar way on the two plates, there are some intrinsic differences between the two: for instance, the amplitude of the deformation tends to be higher at the centre of the CCD on plate 1; the standard deviation of the 'perturbation', $\sigma_{2}$, tends to be offset to lower values.

Therefore, even if it is reassuring to observe a similar behaviour in the evolution of the deformation of the LSF during the night, it seems preferable to determine a LSF model on the calibration lamp of each observation run. Using a global LSF model for all the observations done on a single plate during a single night nevertheless yields acceptable final results, even though the uncertainty of the derived radial velocities tends to be $20-30 \%$ higher than when using one LSF model for each calibration lamp.

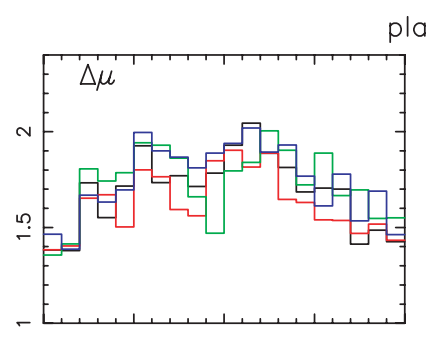

plate 0
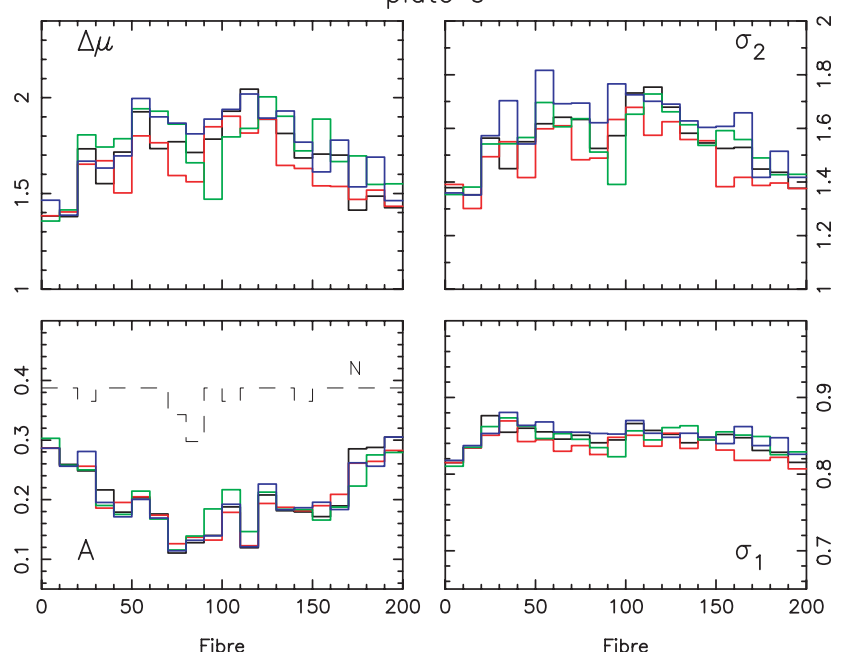

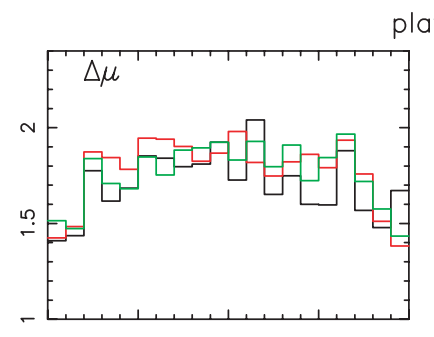

plate 1
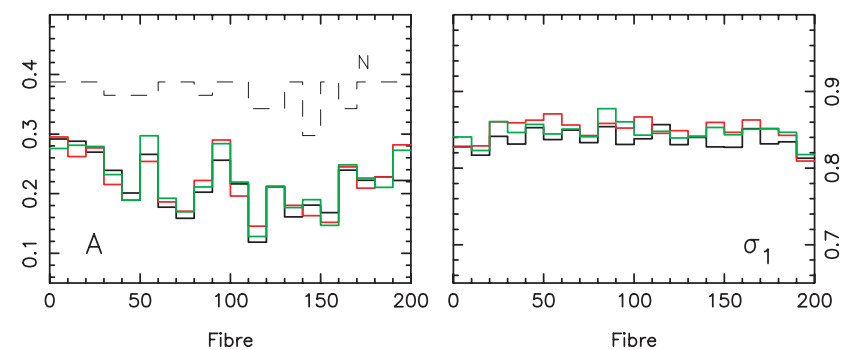

Figure 4 Same as Figure 3 but with the evolution of the LSF parameters for all the observations that were made during a single night. The parameters for observations on plate 0 are shown in the left panels and those performed on plate 1 are shown in the right panels. Although the parameters evolve in a similar way for all the fits, there are variations with plate and/or observation that prevent us from using a generic set of parameters for the whole night. 


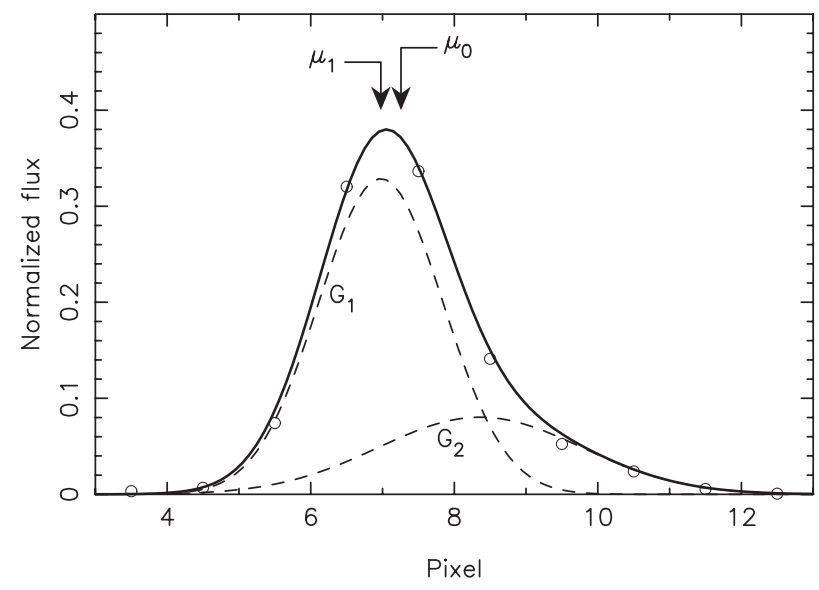

Figure 5 Difference in the central position of an EL for two calibration methods. $\mu_{0}$ is defined as in Equation (10) and corresponds to the value given by the IRAF routine IDENTIFY while $\mu_{1}$ is the centre of the 'theoretical signal', $G_{1}$, of the LSF, as defined in Equation (6). The observed values, $F$, of the EL are shown as hollow circles, while the LSF model, $\widetilde{F}$, determined following the procedure of Section 2.2 is represented as a thick line. Its two components (the 'theoretical signal' $G_{1}$ and the 'perturbation' $G_{2}$ ) are plotted as thin dashed lines. In this example, there is a shift of 0.28 pixels between the centres of the LSF, $\mu_{0}$, and $\mu_{1}$, determined by the two different methods.

\subsection{Calibration}

One part of the reduction where the asymmetry of the LSF has an important effect is the calibration of the spectra. Indeed, in a routine like IDENTIFY in IRAF the centre, $\mu_{0}$, of an emission line $\widetilde{F}$ defined over the range $\left[X_{\min }, X_{\max }\right]$ is the wavelength at which the EL can be divided into two equally luminous parts, that is

$$
\int_{X_{\min }}^{\mu_{0}} \widetilde{F}(X) \mathrm{d} X=\int_{\mu_{0}}^{X_{\max }} \widetilde{F}(X) \mathrm{d} X .
$$

Hence, the presence of the asymmetry in the EL tends to switch the 'IRAF centre' $\mu_{0}$ to higher values than the 'theoretical centre' $\mu_{1}$ which should be considered as a more valid position of the EL (see Figure 5). With the evolution of this offset directly related to the LSF parameter $A$, the difference $\mu_{0}-\mu_{1}$ changes with the fibre. And since we cross-correlate the observed spectra with templates that we generate (see the next subsection) and do not have this calibration issue, the deduced radial velocity can have a systematic error of up to $\sim 10 \mathrm{~km} \mathrm{~s}^{-1}$.

To overcome this effect, we have to calibrate each arc spectrum using the LSF shape of the corresponding fibre, time and plate to determine the position of the 'theoretical' EL $\left(\mu_{1}\right)$ instead of the centre of the asymmetric, observed EL $\left(\mu_{0}\right)$. For a given fibre of a calibration lamp, we proceed as follows:

(1) For each EL of the corresponding fibre on the calibration lamp, its 'theoretical centre' $\mu_{1}$ is determined by fitting the EL model of that fibre (Equation (7)) with $\mu_{1}$ as the only free parameter (an example is given Figure 5).

(2) All the 'theoretical centres' are fitted with a Legendre polynomial (about ten reliable ELs are used for this

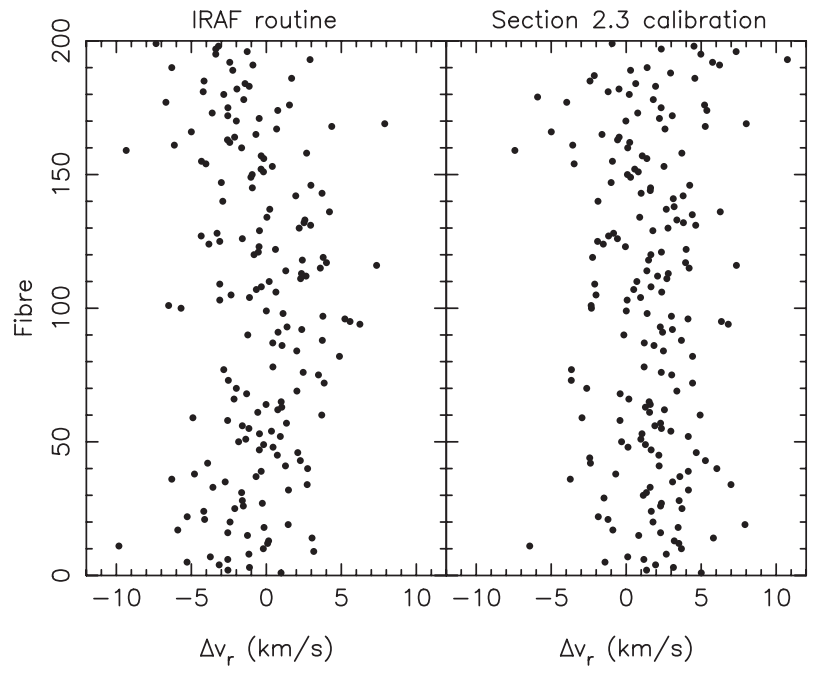

Figure 6 Difference in radial velocity between the different fibres and fibre 100 for a twilight observation. In the left panel, the spectra were calibrated using the IRAF routine IDENTIFY; in the right panel the calibration was performed using the shape of the LSF, as explained in Section 2.3. The IRAF calibration generates an arc-shaped systematic error that depends on the fibre number while our procedure does not introduce such errors.

fit) to determine the wavelength of each pixel of the observed spectrum.

(3) The spectrum is re-binned so that each pixel is $1 \AA$ wide, starting at $4600 \AA$ and ending at $5600 \AA$.

Using the 'theoretical centres' to calibrate the spectrum ensures a calibration that is less altered by the shape of the $2 \mathrm{dF}$ LSF. Indeed, though we do not take into account possible evolutions of the LSF asymmetry along the wavelength direction of the CCD — we fit the same LSF model to all ELs - this technique gives a much better calibration than when using the simpler IRAF routine. To test that assumption one can measure the difference in radial velocity for the different fibres of a twilight observation. Since in such an observation, the spectrum on each fibre is that of the Sun, cross-correlating each calibrated spectrum with a reference spectrum (say, the one of fibre 100) directly reveals calibration-related systematic errors. This test was done for twilight observations of 2004 April 9 and the difference in radial velocity between the different fibres is shown in Figure 6 for a calibration using the IRAF IDENTIFY routine (left) and the procedure described above (right).

It is clear that directly using the IRAF routine is not satisfactory with a velocity difference between fibre 100 and the other fibres that is not constant and which follows an arc with increasing discrepancies at the edge of the CCD. On the other hand, this effect is corrected when using our calibration procedure and all the fibres produce a similar radial velocity, with a small dispersion of $2.8 \mathrm{~km} \mathrm{~s}^{-1}$.

\subsection{Generating Templates}

Measuring the radial velocity of a star requires one to cross-correlate the stellar spectrum with that of a template 
star of known radial velocity. Generally, a template spectrum is obtained by observing, along with the target stars, a few well-known stars. However, due to time constraints, one is forced to observe a small number of templates during a specific night, with a specific fibre of the $2 \mathrm{dF}$, whereas as we saw earlier, the asymmetry of the LSF can evolve with time and certainly changes with the fibre (see Figure 4). Consequently, the cross-correlation would be produced by two spectra deformed in different ways, which can produce systematic errors on the measured shift between the two spectra of the order of 0.2 pixels (corresponding to as much as $15 \mathrm{~km} \mathrm{~s}^{-1}$ on CCD1). Hence, it is necessary to take the LSF shape of the templates into account when doing the cross-correlation.

Since it is cumbersome to deconvolve the spectrum of the templates and then reconvolve it with the LSF of the fibre one is interested in, we choose to generate artificial templates by convolving high-resolution (HR) spectra by the deformed LSF. This way, the target and template spectra are similarly deformed and their cross-correlation no longer has systematic effects due to differences in the deformation of the LSF of the two spectra. For each group of ten fibres, we proceed with the following steps:

(1) convolution of the HR template with the corresponding LSF (as defined in Equation (6) and determined as explained in Section 2.2);

(2) downgrading the HR, convolved template to a low resolution, 2dF-like template ( $1 \AA$ per pixel for the $1200 \mathrm{~V}$ grating);

(3) cross-correlation of the observed spectra with the artificial template using the FXCOR routine in IRAF.

We choose the HR spectra from the UVES Paranal Observatory Project ${ }^{6}$ (Bagnulo et al. 2003) which has the advantage of providing the spectrum of stars of numerous spectral types (from type $\mathrm{O}$ to type $\mathrm{M}$ stars) and hence provides the opportunity to choose a template that resembles the observed stars. Moreover, the UVES observations made through the $580 \mathrm{~L}$ grating (4760-5770 ̊) correspond to the range we are interested in (4800-5250 ̊).

\section{$2.5 C C D 2$}

As is visible in Figure 7, the calibration lamps observed on CCD2 show emission lines that do not have asymmetries like CCD1. In fact, the emission lines are well fitted by a single Gaussian model (line in Figure 7) with uncertainties on the position and dispersion of the Gaussian lower than 0.01 pixel. It is therefore not necessary to apply the previous reduction techniques to obtain precise radial velocities from observations on CCD2. However, the symmetry of the emission lines does not mean that there are no issues with this CCD. Indeed, analyzing the same EL for all the fibres (the 8408.2096 $\AA \mathrm{CuAr}$ line roughly located at the centre of the $\mathrm{CCD}$ ) shows that there

\footnotetext{
${ }^{6}$ www. sc.eso.org/santiago/uvespop/index.html
}

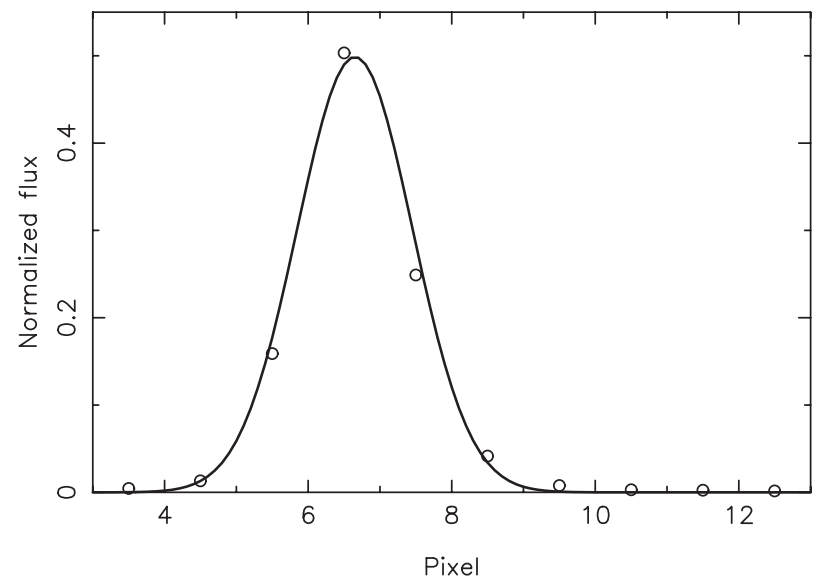

Figure 7 8408.2096 A CuAr emission line extracted from fibre 1 of a CCD2 calibration lamp observation (circles). The emission line is symmetric and can be fitted by a single Gaussian function of internal dispersion $\sigma=0.74$ (line).

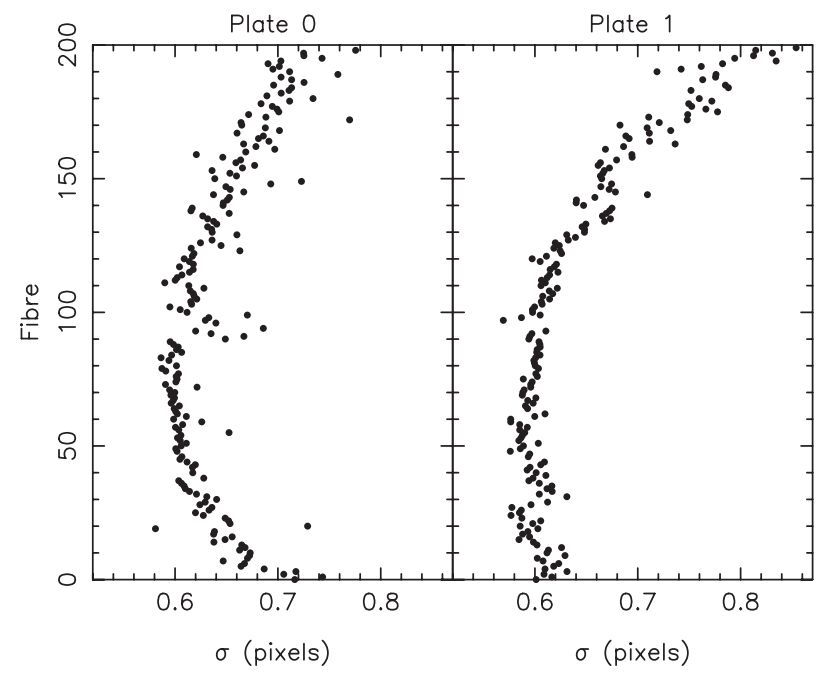

Figure 8 Evolution of the internal dispersion, $\sigma$, of the LSF across CCD2 when fitted by a single Gaussian function as in Figure 7. Although the LSF remains symmetric across CCD2 (contrary to CCD1), its width varies substantially.

are substantial changes in the internal dispersion, $\sigma$, of the Gaussian fit. Once again, $\sigma$ has an arc-like evolution across the CCD (see Figure 8). Moreover, the evolution differs with the plate on which the observations were performed. Even though an increase in the dispersion is expected at the edges of the CCD, the magnitude of the variation (from $\sigma=0.60$ at the centre up to $\sigma=0.75$ at the edge of plate 0 observations or $\sigma=0.85$ at the edge of plate 1 observations) is troublesome. In addition, there is at least one group of fibres (the 90-100 group on plate 0) that has a higher internal dispersion than its neighbouring groups.

Although the symmetry of the ELs means that the 2DFDR reduction package should suffice to reduce the data precisely, the variation of the internal dispersion of the LSF on CCD2 is to be kept in mind if some systematic effects remain at the end of the reduction. 

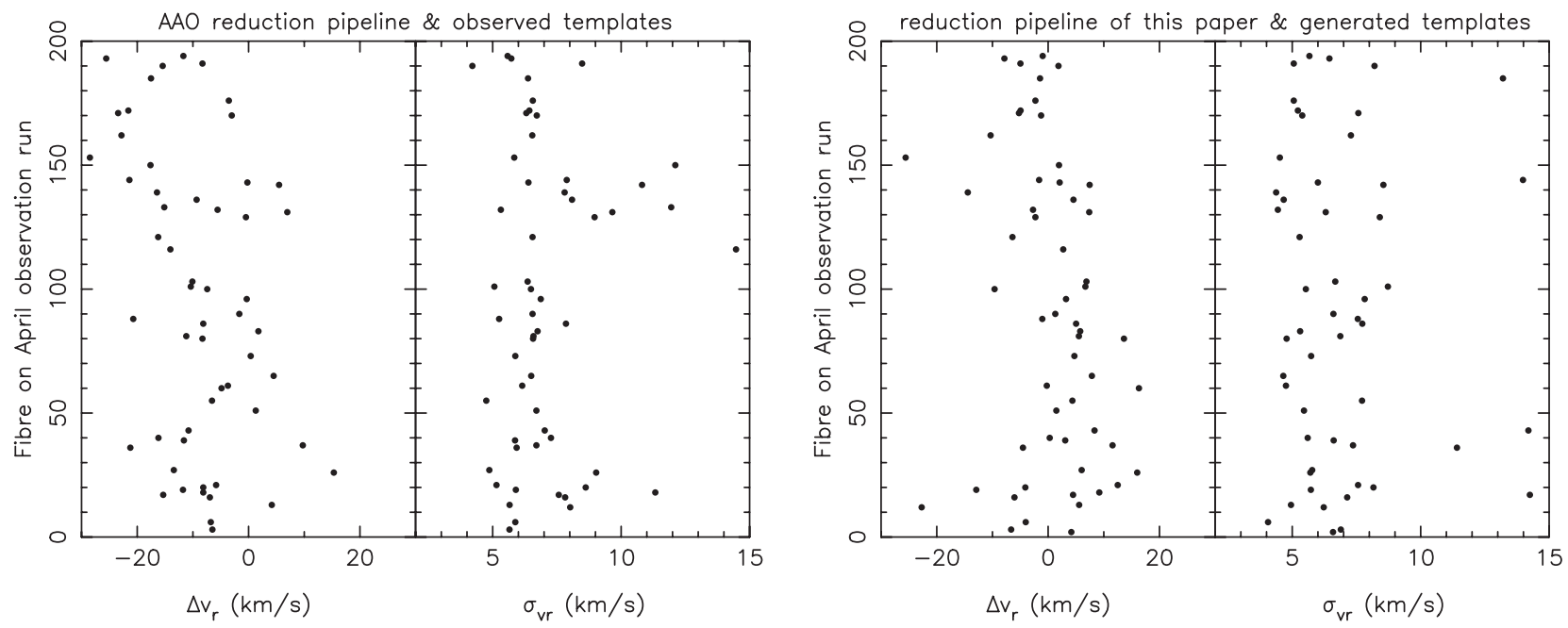

Figure 9 Difference in radial velocities between the April and December observations when reducing the data using the 2DFDR package (left panels) and the reduction pipeline presented in this paper (right panels). For each set of panels, the offset in radial velocity, $\Delta v_{r}=v_{r, \mathrm{Apr}}-v_{r, \mathrm{Dec}}$, has been plotted (left) along with the uncertainties on these measurements (right). Using the reduction techniques developed in this paper produces lower systematic errors with $\Delta v_{r} \sim 0 \mathrm{~km} \mathrm{~s}^{-1}$ and a lower internal dispersion of the offsets.

\section{Results}

To analyze the efficiency of the procedure we present here, we observed one of the fields of our CMa survey twice. The first observation run was performed on 2004 April 9, using the spectrograph 1 settings (CCD1 and $1200 \mathrm{~V}$ grating; this is our April dataset) and the second run was performed on 2004 December 3, this time using spectrograph 2 settings (CCD2 and $1200 \mathrm{R}$ grating; this is our December dataset). As we showed, the radial velocities derived from the December observations on CCD2 should not have important systematic effects. Moreover, the use of the $1200 \mathrm{R}$ grating produces low uncertainties on the velocities $\left(\sim 2 \mathrm{~km} \mathrm{~s}^{-1}\right)$. In the following, we will use the December radial velocities as a reference and analyze the influence of our pipeline on the April radial velocities.

When using the 2DFDR package to reduce the April observations (left panels of Figure 9), the radial velocities are broadly $\sim 10-20 \mathrm{~km} \mathrm{~s}^{-1}$ lower than the reference velocities measured in the December run, but some stars have offsets as low as $-30 \mathrm{~km} \mathrm{~s}^{-1}$ or as high as $20 \mathrm{~km} \mathrm{~s}^{-1}$. At the same time, the uncertainties on these velocities are mainly in the range $6-8 \mathrm{~km} \mathrm{~s}^{-1}$ with, here as well, groups of outliers with uncertainties increasing to $\sim 15 \mathrm{~km} \mathrm{~s}^{-1}$ on different portions of the CCD (for low fibres or fibres between 130 and 150).

In contrast, the reduction of the April observations on CCD1 using the pipeline described in this paper (right panels of Figure 9) yields lower offsets compared to the reference velocities. The mean offset for all the CCD has changed from $-9 \pm 10 \mathrm{~km} \mathrm{~s}^{-1}$ to $0 \pm 8 \mathrm{~km} \mathrm{~s}^{-1}$ and there are only a few outliers $(\sim 2)$. Similarly, the uncertainties on each radial velocity are slightly lower and are more clustered, with no more groups of fibres producing outliers with high uncertainties.

Though the use of the reduction pipeline described in this paper produces much more reliable radial velocities, low systematic offsets still remain. On average, this effect is limited $\left(\sim 5 \mathrm{~km} \mathrm{~s}^{-1}\right.$ and certainly less than $\left.10 \mathrm{~km} \mathrm{~s}^{-1}\right)$ and could either be due to the reduction pipeline that may not completely correct the LSF asymmetry effects (but it has been shown that there are no more calibration-related issues) or be due to systematic errors in the reference velocities. We have shown that the emission lines observed through CCD2 do not present any asymmetry but what remains unclear is the role of the variations of the width of the LSF of CCD2 (see Figure 8) on the radial velocities.

\section{Summary}

We have constructed an improved reduction pipeline for $2 \mathrm{dF}$ observations. The systematic offsets that appeared between radial velocities measured from CCD1 data and those measured from CCD2 data can now be corrected. It has been shown that they are due to an asymmetric line spread function, which for the data we analyzed, particularly affects CCD1. This effect can be countered by

1. modelling the asymmetric LSF by the sum of two Gaussian functions - the first would represent the LSF of the system if it were perfect and the second one is a perturbation of this system;

2. fitting the LSF model to emission lines of the calibration lamps for each group of ten fibres;

3. using this LSF model to calibrate the corresponding spectra;

4. using this LSF model to downgrade high-resolution template spectra so that the observations can be crosscorrelated with templates similarly deformed.

The difference in radial velocity in a set of Red Giant Branch stars that has been observed twice (once on each $\mathrm{CCD}$ ) is highly reduced when using this procedure $\left(\sim \pm 5 \mathrm{~km} \mathrm{~s}^{-1}\right)$. Moreover, the radial velocity 
measurements show a more consistent behaviour throughout the CCD than when using only the AAO reduction pipeline.

Even though the $2 \mathrm{dF}$ spectrograph is to be decommissioned at the end of this year, archival data from the past decade could be improved by the reduction procedure we propose here. In particular, studies of the kinematics of galactic populations could benefit highly from a reduction of the internal dispersion of the radial velocity datasets. The pipeline has only been shown to work for a particular dataset, yet we suspect that observations obtained through other gratings or with other central wevelengths and that present the same kind of systematic effects can be corrected in a similar way.

\section{Acknowledgment}

We would like to thank Robert Sharp for useful comments on this paper.

\section{References}

Bagnulo, S., Jehin, E., Ledoux, C., Cabanac, R., Melo, C., Gilmozzi, R., \& The ESO Paranal Science Operations Team. 2003, The Messenger, 114, 10

Cannon, R. 2002, AAO Newsletter No. 101, p 22

Lewis, I. J., et al. 2002, MNRAS, 333, 279

Martin, N. F., Ibata, R. A., Conn, B. C., Lewis, G. F., Bellazzini, M., Irwin, M. J., \& McConnachie, A. W. 2004, MNRAS, 355, L33

Martin, N. F., Ibata, R. A., Conn, B. C., Lewis, G. F., Bellazzini, M., \& Irwin, M. J. 2005, MNRAS, in press

Press, W. H., Flannery, B. P., Teukolsky, S. A., \& Vetterling, W. T. 1992, Numerical Recipes (Cambridge: Cambridge University Press)

Stanford, L., \& Cannon, R. 2002, 2dF Technical Note, www. aao.gov.au/2df/technotes/fibvel.ps.gz

Taylor, K., Bailey, J., Wilkins, T., Shortridge, K., \& Glazebrook, K. 1996, ADASS, 5, 195 